\title{
Reducing Handover Latency of PMIPv6 using Extended Open-Flow Technique
}

\author{
L. K. Indumathi
}

\begin{abstract}
This proposed technique adapts PMIPv6 to the Extended Open-Flow architecture, and this technique is referred to as the Extended Open-Flow Technique of PMIPv6 (EOFT-PMIPv6). This method isolates the versatility capacities from the PMIPv6 segments, for example, the Local Mobility Anchor (LMA) and Mobile Access Gateway (MAG), and recreates the parts to take points of interest of the Open-Flow design. The parts that contain the LMA work set the stream table of the switches situated in the way as the controller of Open-Flow, and as such, the area of the MN is kept up. The entrance switches with the MAG capacities tell the connection of a MN and introduce the portability related motioning of MAG. The fundamental commitments of this proposed strategy are twofold: 1) isolating the control and data planes and 2) reducing handover latency.
\end{abstract}

KeyWords: Control Plane, Data Plane, Local Mobility Anchor (LMA), Mobile Access Gateway (MAG), Proxy Mobile Ipv6 (PMIPv6), Open-Flow (OF).

\section{INTRODUCTION}

Software-Defined Networking (SDN) is an emerging architecture that is dynamic, manageable, cost-effective, and adaptable, making it ideal for the high-bandwidth, dynamic nature of today's applications. The Open-Flow convention is a basic component for building SDN arrangements [1]. Open-Flow Technique (OFT) is a cutting edge innovation to advance the directing way by applying the Open-Flow procedure in PMIPv6. The principle highlight of the Open-Flow strategy is to isolate crafted by system gadgets. The system gadgets forward parcels utilizing control and information capacities.

Hampel et al. [2] proposed a product characterized organizing for telecom area, where this strategy was effectively actualized in server farms and grounds arranges in any case, it has had little effect on the settled wire line and portable telecom space. Devarapalli [3] proposed a strategy for sending of PMIPv6 by isolated control and information plane end focuses for the MAG. There exists a different IP address for the element that sends and gets the Proxy Mobile IPv6 flagging messages. Likewise, there is a different IP address for the substance that epitomizes and decapsulates the information traffic to/from the versatile hub. According to Gundavelli [4]) and Johnson et al. [5] the LMA stores IP address, the Proxy Care-of Address (PCoA), in the proxy binding cache entry at the LMA. Therefore, the LMA uses the same IP address of a MAG for signaling messages and data traffic. Wakikawa et al. [6] this document explains a

Revised Manuscript Received on 14 August, 2019.

Dr.L.K.Indumathi, Professor, Department of Computer Science and Engineering, Mallareddy Engineering College for Women, Hyderabad, India. induchunku@gmail.com mechanism to separate the Control Plane (CP) and User Plane (UP) from a network infrastructure based on PMIPv6. According to Lee et al. [7], there have been a significant number of methodologies for versatility the board in IP systems to give session coherence to portable hubs bridging heterogeneous remote systems. Kim et al. [8] proposed a strategy with Open-Flow-based Proxy portable IPv6 in SDN in adaptable system. PMIPv6 is proposed to deal with the system based nearby portability utilizing IP burrowing, however the confinement of this strategy is burrowing overhead and having a similar way for the information and the control planes.

\section{PROPOSED WORK Configuration EOFT-PMIPV6}

PMIPv6 signaling can be excluded as a result of the co-location of the LMA and the MAG functions in the EOFT-Controller as shown in Fig.1.

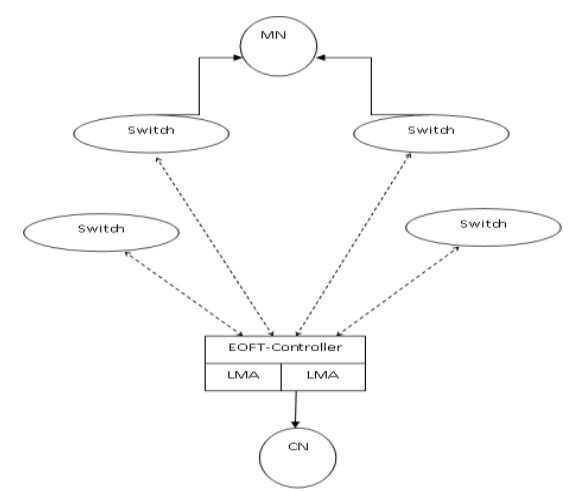

Figure 1. Configuration of Control plane in EOFT-PMIPv6 EOFT-PMIPv6 uses only the extended Open-Flow messages that support purposes i) switching forwarding packets based on the policies ii) making a controller to configure flow tables and switches and then the extended Open-Flow messages are forwarded to the LAM at the MAG modules. The information related to 1) and 2) is processed by using an internal interaction between those functions.

The MAG in PMIPv6 detects the attachment and detachment of the MN. The detection is initiated by the link layer that establishes the connection. The link layer state information is maintained by the MAG system, and the MAG function detects an attachment based on the link layer state, which is provided by the MAG system, as assumed in the PMIPv6 standard. In EOFT-PMIPv6, the link layer state is forwarded to the MAG function located in the controller in order to detect the establishment of a link layer connection in the same manner as PMIPv6. The switch sends the link layer state by using the extended Open-Flow messages with the mobility options such as the MAG address and the access technology of the MN, and doing so is a simple procedure. Thus, the MAG function on the controller can detect an 
attachment and detachment of an MN.

The data path for EOFT-PMIPv6 is configured like OPMIPv6 but here, both the LMA and the MAG controllers are kept in the EOFT-Controller, as shown in Fig.4. When the movement of the MN is detected with the help of PMIPv6 signaling, the LMA and MAG controllers update the flow table of the intermediate switches on the path between the access router that detected the movement and the gateway. Hence packet forwarding does not use an IP tunnel in EOFT-PMIPv6, and the LMA controller can distribute traffic for local balancing, depending on the conditions of the network according to its configuration.

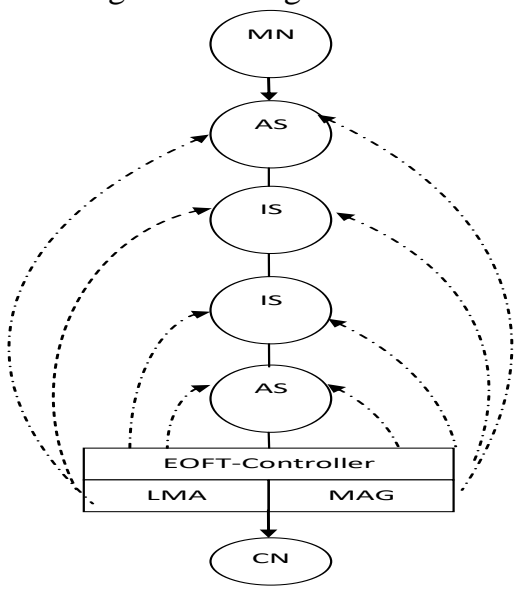

Fig. 4. Packet forwarding of data plane in EOFT-PMIPv6

For the evaluation, the proposed technique uses network model and concept similar to those presented in reference Lee et al. [9]. The topology is shown in Fig. 5, and it is used to represent the provisioning entities in PMIPv6, OPMIPv6, and EOFT-PMIPv6.

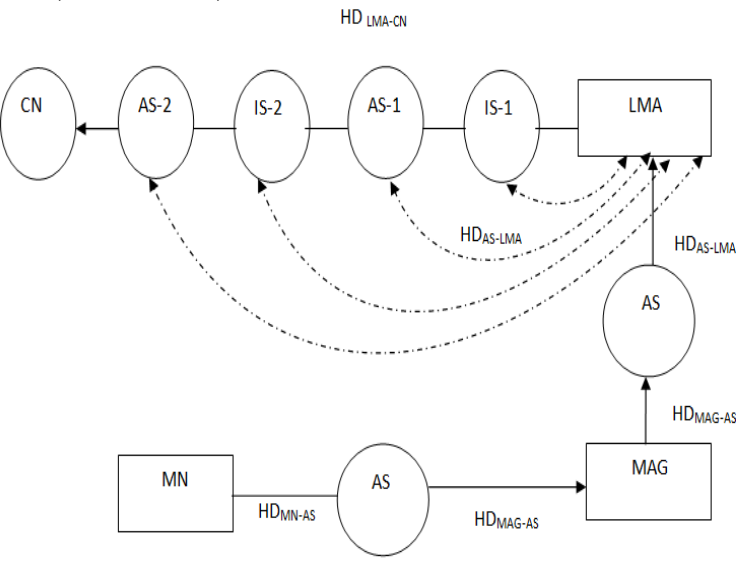

Figure 5. Topology of EOFT-PMIPv6

The gateway and the Access Switch (AS) respectively work as the LMA and the MAG in PMIPv6. For OPMIPv6, the gateway can work as a controller with LMA functionality, and the AS works as an Open-Flow switch with MAG functionality. In EOFT-PMIPv6, the gateway acts as the controller with the LMA and the MAG function, and the AS only acts as the Open-Flow switch. It is assumed that the gateway also has its own Open-Flow switch, and several Intermediate Switches (ISs) are located between the gateway and the ASs. The $\mathrm{CN}$ is placed outside a given administrative domain, and the movement of the MNs is limited in the domain where the gateway performs as a border router. HDLMA-CN: Hop distance between the LMA and CN. HDAS-LMA: Hop distance between the AS and LMA.
HDMN-AS: :Hop distance between the MN and AS. HDMAG-AS: Hop distance between the MAG and AS.

As shown in Figure 5, the HDAS-LMA indicates the hop distance of a secure channel between the gateway (LMA and MAG controller) and the switches (IS and AS) for Open-Flow signaling. It is assumed that the secure channel is one hop away in distance. In order to develop the mobility model, the session arrival process for the $\mathrm{MN}$ is assumed to follow a Poisson distribution with a rate $\chi_{\mathrm{s}}[10]$.

\section{A. Simulation Description Of EOFT-PMIPv6}

According to simulation topology, the default values of the system parameters for the cost analysis of EOFT-PMIPv6 are set. The simulation is run for 30 seconds. The mobility session speed is $100 / \mathrm{mbps}$. Also, the various interfaces appear in the simulation in various seconds. According to the references [4],[11] parameters are fixed for the simulation .

\section{HANDOVER ANALYSIS OF PROPOSED WORK}

\section{A) Handover Analysis of PMIPv6}

According to signal flow of PMIPv6 [4] the handover is analyzed. The MN is moving from one MAG to another. The localized routing with its $\mathrm{CN}$ and the Hand Over (HOD) are calculated in the following equation. $\mathrm{HOD}_{\mathrm{PMIPV} 6}=$ Layer 2 connection $+(\mathrm{tPBA}+\mathrm{tPBU})+\mathrm{tRS}+$ tRA+ (tAAAreq + tAAAres) + TTD Data -----------( 1)

Here, Layer 2 connection represents transmission delay between MN-AP and transmission delay between AP-MAG. The delay of control signal is represented as $(\mathrm{tPBU}+\mathrm{tPBA})$, and authentication delay is represented as tAAAreq + tAAAres.

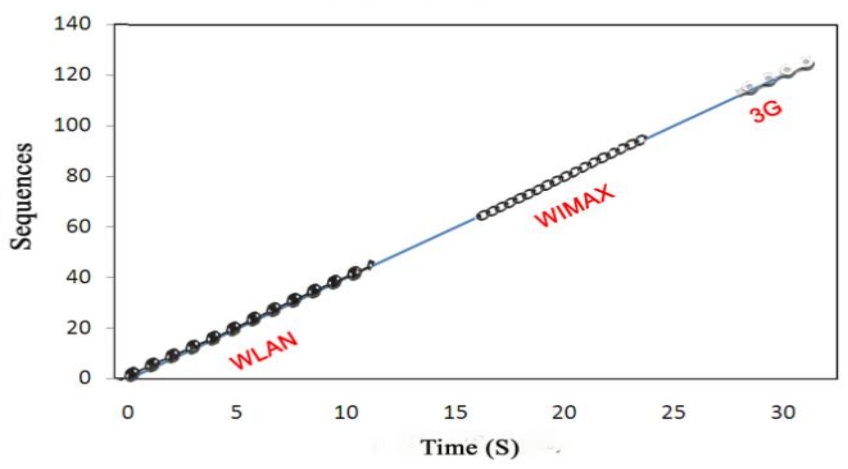

Fig. 6. Handover Simulation result of PMIPv6

In the simulation, the MN starts with its current interface, i.e. with WLAN. Wi-Max enters at 11th second in the simulation, but the MN hands over its signal to Wi-Max at 13.9 seconds. $3 \mathrm{G}$ enters at 24.5 th second in the simulation, but the $\mathrm{MN}$ hands over its signal to $3 \mathrm{G}$ at 28 th second. Figure 6 depicts handover graph of PMIPv6.

\section{B) Handover Analysis of O-PMIPv6}

O-PMIPv6 uses PMIPv6 signaling and Open-Flow signaling. It is assumed that Open-Flow signaling finishes the ISs before the PBA message reaches the AS. This is because Open-Flow signaling is practically performed through secure channel and the distance of the AS is the longest. Thus, the handover latency for OPMIPv6 is similar to that of PMIPv6. However, HODOMPIV6 is 
different from HODPMIPv6 because the data packets are forwarded without an IP tunnel in OPMIPv6. Thus, it can be expressed as follows.

HOD $_{\text {O-MPIv6 }}=$ Layer 2 connection +2 (TTDData $)+$ HDMAG-AS-

Here, Layer 2 connection represents Transmission Delay between MN-AP and Transmission delay between AP-MAG. 2 (TTDData) represents transmission time of data for two times because for separate LMA and MAG details of Open-Flow controller.

\section{B. Handover simulation result of OPMIPv6}

In the simulation, the MN starts with its current interface, i.e. with WLAN and WLAN handover to Wi-Max at 11th second, but MN enters Wi-MAX at 13.1 seconds and Wi-Max handover to $3 \mathrm{G}$ at 24.5 seconds, but the MN enters $3 \mathrm{G}$ at 26th second. Fig.7. shows the handover simulation result of OPMIPv6.

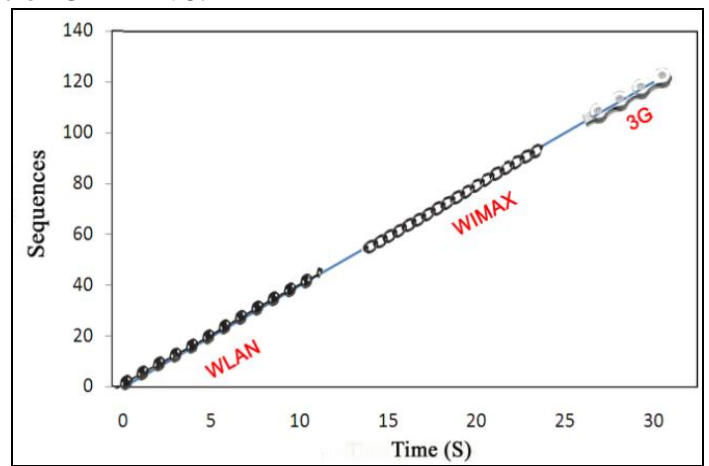

Figure.7. Handover simulation result of $O-P M I P v 6$

\section{C) Handover Analysis of EOFT-PMIPv6}

EOFT-PMIPv6 also uses both Open-Flow signalling and PMIPv6 signaling performed through a secure channel with extended architecture over TCP. HODOPMIPV6 is different from HODEOFT-PMIPV6 because the propagation time of the secure channel is assumed to proportionally increase according to the distance between the MAG-AS. Also, in EOFT-PMIPv6 both LMA and MAG details are maintained simultaneously with the EOFT-Controller. So, the HODEOFT-PMIPV6 is expressed as follows

HODEOFT-PMIPv6 = Layer 2 connection + (TTDData $)+$ HDMAG-AS-------------------(3)

\section{Handover simulation result of EOFT-PMIPv6}

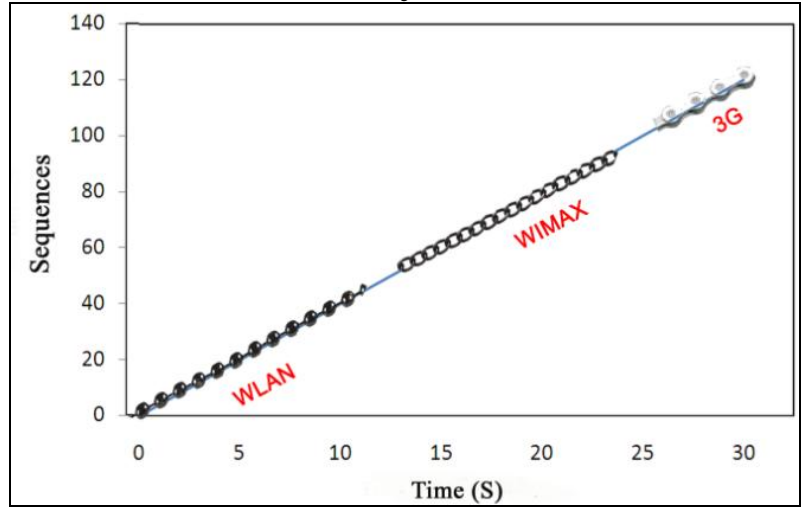

Figure.8. Handover Simulation result of EOFT-PMIPv6

In the simulation, the $\mathrm{MN}$ starts with its current interface, i.e. with WLAN. Wi-Max enters at 11th second in the simulation, but the MN hands over its signal to Wi-Max at 11.5 seconds. $3 \mathrm{G}$ enters at 24.5 th second in the simulation, but the $\mathrm{MN}$ hands over its signal to $3 \mathrm{G}$ at 25 th second. Fig.8 shows the of handover simulation result of EOFT-PMIPv6.

\section{D) Comparative Analysis of Handover Latency of EOFT-PMIPv6}

The handover latencies of EOFT-PMIPv6 are compared with PMIPv6/SAND, OPMIPv6 and EOFT-PMIPv6 and the results are graphically depicted in Fig.9. The handover latency of PMIPv6 is observed to be the highest because it does not use Open-Flow signaling. The handover latency of OPMIPv6 is higher than that of EOFT-PMIPv6 because it has separate Open-Flow controllers for LMA and MAG. Thus, the handover latency of EOFT-PMIPv6 has the least latency compared to other techniques.

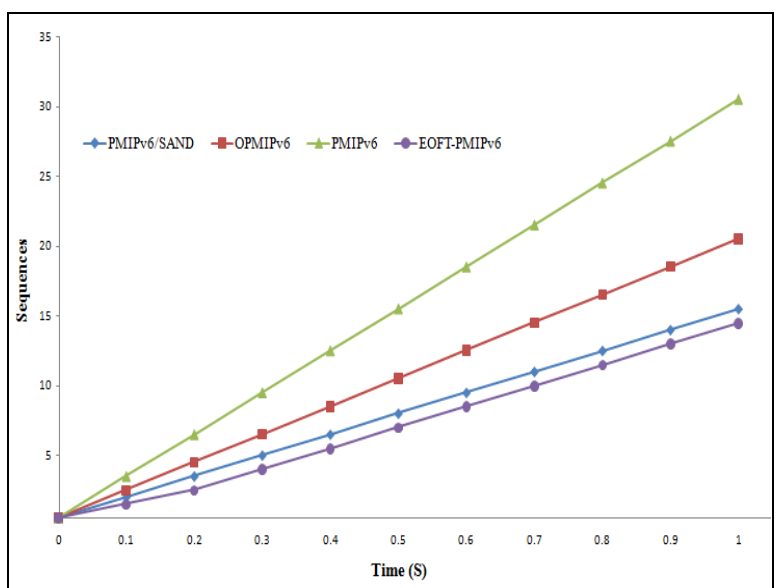

Figure. 9. Comparative Analysis of Handover Latency of EOFT-PMIPv6

\section{CONCLUSION}

EOFT-PMIPv6 is an adaptation of PMIPv6 to the open flow architecture. In order to support mobility in the Open-Flow architecture, the mobility functions are separated from the PMIPv6 entities and are located in the controller and the switches. Also, this proposed technique provides a more flexible configuration architecture that can increase the handling capacity and can resist failure through its EOFT-Controller. The results of the performance evaluation indicate that EOFT-PMIPv6 is more efficient than PMIPv6 and slightly improves its performance.

\section{REFERENCES}

1. Denicol, RR, Fernandes, EL, Rothenberg, CE \& Kis, ZL 2011, 'On IPv6 support in Open-Flow via flexible match structures', J. OFELIA/CHANGE Summer School.

2. Hampel, G, Steiner, M \& Bu, T 2013, 'Applying software-defined networking to the telecom domain', In Computer Communications Workshops (INFOC OM WKSHPS), IEEE Conference on IEEE, pp. 133-138.

3. Devarapalli, V 2008, 'Separating Control and Data Plane for Proxy Mobile IPv6'.

4. Gundavelli, S, Leung, K, Devarapalli, V \& Chowdhury, K 2008, 'B. Patil', Proxy Mobile IPv6. RFC 5213.

5. Johnson, D, Perkins, C \& Arkko, J 2004, 'Mobility support in IPv6 (No. RFC 3775)',

6. Wakikawa, R, Pazhyannur, R, Gundavelli, S \& Perkins, C 2014, 'Separation of Control and User Plane for Proxy Mobile IPv6 (No. RFC 7389)'.

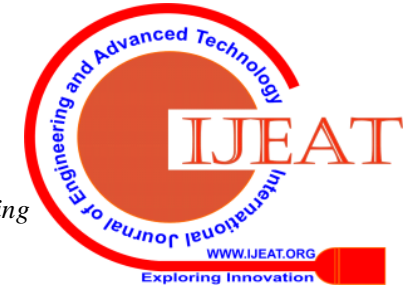


7. Lee, KH, Mougouei, D, Yeung, MK, Park, HJ, Bae, JH \& Hameed, A 2014, 'Mobility management framework in software defined networks', International Journal of Software Engineering and Its Applications, vol. 8, no. 8, pp. 1-10.

8. Kim, SM, Choi, HY, Park, PW, Min, SG \& Han, YH 2014, 'Open-Flow-based Proxy mobile IPv6 over software defined network (SDN)’, In 2014 IEEE 11 th Consumer Communications and Networking Conference (CCNC) IEEE, pp. 119-125.

9. Lee, JH, Ernst, T \& Chung, TM 2010, 'Cost analysis of IP mobility management protocols for consumer mobile devices', IEEE Transactions on Consumer Electronics, vol. 56, no. 2, pp. 1010-1017.

10. Baumann, FV \& Niemegeers, IG 1994, 'An evaluation of location management procedures. In Universal Personal Communications’, 1994. Record, 1994 Third Annual International Conference on IEEE, pp. 359-364.

11. Specification-Version, Open-Flow Switch. "1.4. 0." (2013) 